Article

\title{
Enhanced Feature Subset Selection Using Niche Based Bat Algorithm
}

\author{
Noman Saleem ${ }^{1}$, Kashif Zafar ${ }^{2, *}$ and Alizaa Fatima Sabzwari ${ }^{2}$ \\ 1 Department of Software Engineering, the University of Lahore, Lahore 54590, Pakistan; \\ noman.saleem.4@gmail.com \\ 2 National University of Computer and Emerging Sciences, Lahore 54770, Pakistan; alizaa.fatima7@gmail.com \\ * Correspondence: kashif.zafar@nu.edu.pk
}

Received: 10 April 2019; Accepted: 6 June 2019; Published: 6 September 2019

\begin{abstract}
Redundant and irrelevant features disturb the accuracy of the classifier. In order to avoid redundancy and irrelevancy problems, feature selection techniques are used. Finding the most relevant feature subset that can enhance the accuracy rate of the classifier is one of the most challenging parts. This paper presents a new solution to finding relevant feature subsets by the niche based bat algorithm (NBBA). It is compared with existing state of the art approaches, including evolutionary based approaches. The multi-objective bat algorithm (MOBA) selected 8, 16, and 248 features with $93.33 \%, 93.54 \%$, and $78.33 \%$ accuracy on ionosphere, sonar, and Madelon datasets, respectively. The multi-objective genetic algorithm (MOGA) selected 10, 17, and 256 features with $91.28 \%, 88.70 \%$, and $75.16 \%$ accuracy on same datasets, respectively. Finally, the multi-objective particle swarm optimization (MOPSO) selected 9, 21, and 312 with $89.52 \%, 91.93 \%$, and $76 \%$ accuracy on the above datasets, respectively. In comparison, NBBA selected 6, 19, and 178 features with $93.33 \%$, $95.16 \%$, and $80.16 \%$ accuracy on the above datasets, respectively. The niche multi-objective genetic algorithm selected 8, 15, and 196 features with $93.33 \%, 91.93 \%$, and $79.16 \%$ accuracy on the above datasets, respectively. Finally, the niche multi-objective particle swarm optimization selected 9, 19, and 213 features with $91.42 \%, 91.93 \%$, and $76.5 \%$ accuracy on the above datasets, respectively. Hence, results show that MOBA outperformed MOGA and MOPSO, and NBBA outperformed the niche multi-objective genetic algorithm and the niche multi-objective particle swarm optimization.
\end{abstract}

Keywords: feature selection; genetic algorithm; niche; bat algorithm; particle swarm optimization

\section{Introduction}

Feature selection is a method that selects features or a subset of relevant features of a dataset to achieve the best accuracy through a certain classifier. There are two main purposes of feature selection. Firstly, it makes training easier, as applying a classifier is now more efficient because it decreases the size of the dataset by reducing features. This is specifically important for classifiers. Secondly, most of the time, feature selection eliminates noise in the dataset, thus increasing the classification accuracy. A noise feature can increase the classification error of that dataset if it is added. The best feature subset selection has proven to be a nondeterministic polynomial (NP)-complete problem [1]. The task is challenging; initially, the features do not seem relevant when selected individually but seem to be highly relevant when selected with other features. Feature subset selection serves as a pre-processing step for the classification process. The higher the relevance of selected features is, the higher the accuracy of the classifier will be. There are several feature selection techniques that are widely used, such as the two-step protocol that uses F-score for ranking features, as F-score reveals the discriminative power of each feature independently. The features with the highest scores are the most discriminative features, therefore they are selected in the feature subset selection process as the resultant dataset [2-5]. 
Another method for feature selection is the maximum relevancy minimum redundancy (mRMR); this method selects features with higher relevance or maximum correlation while minimizing subsets with higher redundancy that cause the classification accuracy to decrease [6,7]. Feature selection is a costly process, and to solve this, many non-heuristic and heuristic methods have been applied. These methods include statistical methods that use univariate as well as multivariate methods. Multivariate methods provide better results, even when features are dependent on datasets of a particular area or domain. The multivariate methods are further divided into wrapper, filter, and hybrid approaches [8]. The filter approach does the feature subset selection without the involvement of classifiers, while the wrapper approach includes the classifier, which improves the solutions in every iteration and evaluates the accuracy in multiple iterations. This makes the wrapper approach a better candidate for providing a solution [9].

Many meta-heuristic methods have been used for feature selection (FS), which include genetic algorithm (GA), particle swarm optimization (PSO), ant colony optimization (ACO) etc. However, these algorithms propose a computationally expensive solution for feature subset selection. Many computational intelligence based algorithms [10], such as the GA [11] and the PSO [12,13], have been applied for feature selection problems on certain benchmark datasets. The genetic algorithm is a biologically inspired evolutionary algorithm and can achieve a high classification accuracy, but as it is a computationally expensive algorithm, it leads to less efficient and less optimized solutions. On the other hand, PSO provides efficient solution strategies for feature subset selection problems. Similarly, the bat algorithm has been used for feature subset selection problems and gives better results as compared to GA and PSO.

An improved feature selection method for larger datasets is an ongoing research problem. In addition, the existing proposed solutions are either dependent on the classifier or on the dataset. Moreover, the current feature subset selection approaches result in redundant or unrelated features within the search space, and this causes the classifier's performance to decrease. However, selecting related and relevant features correctly can enhance the classifier's performance for a particular dataset. Due to the inconsistencies and the irregularities in the existing methods, the niche based bat algorithm (NBBA) was proposed. It is based on the existing bat algorithm. The k-nearest neighbor (k-NN) [14] algorithm was used as the classifier. Niching is a technique used in multimodal optimization, and it was initially applied on GA [15]. It divides the main population into multiple sub-populations, and each sub-population converges to its local optima. The bat algorithm is inspired by nature, which uses the power of echolocation of micro-bats. Each of the bats symbolize a complete solution, and the classifier is used to evaluate and select the best bat with the highest classification accuracy and the optimum number of feature subsets. Classification accuracy is the fitness of the feature subsets, and in order to evaluate the fitness, a classifier is used. Initially, the bat algorithm (BA) was used in order to optimize the feature subsets, and as this problem is multi-objective in nature, the multi-objective bat algorithm (MOBA) was also tested and compared with the multi-objective genetic algorithm (MOGA) and the multi-objective particle swarm optimization (MOPSO), while the niche based bat algorithm (NBBA) was tested and compared with the niche multi-objective genetic algorithm and the niche multi-objective particle swarm optimization.

\subsection{Motivation for Using Bat Algorithm for This Research}

The BA [16] was chosen because it possesses the advantages of other biologically inspired algorithms, and mainly because it includes the properties of the PSO and the Harmony Search algorithms [17]. BA automatically changes its parameters as the iterations proceed, in contrast to other algorithms that use fixed parameter settings. Another advantage is that BA is more efficient than other algorithms, as it automatically zooms into the promising regions of the search space and converts its search from exploration of those promising regions, which make its convergence rate faster [17]. Combinatorial problems are really hard to solve, and the feature subset selection is an NP-complete combinatorial problem. Ramesh et al. proposed a comprehensive study of combined economic load 
and emission dispatch problems using the bat algorithm [17]. They compared the bat algorithm with other algorithms, including ACO and the hybrid genetic algorithm, concluding that the bat algorithm is better than other algorithms in terms of efficiency, accuracy, and ease of implementation. Musikapun and Pongcharoen solved multi-product, multi-machine, and multi-stage scheduling problems using the bat algorithm [18], and they successfully solved a class of NP-hard problems with a detailed parametric study. Therefore, the existing work indicates the reason for selecting BA for optimization of features in the dataset. The bat algorithm is currently being used to solve various kinds of problems, and fortunately it is very adaptive to the new problems, as researchers are creating newer versions of the bat algorithm that fit their needs [19] The objective functions for the algorithm are to select the minimum relevant features of the dataset and to improve the accuracy of the k-nearest neighbor classifier; all of this combined is a complete solution. The experiments were carried out on benchmark datasets using single, multi-objective, and niche based genetic algorithms, single, multi-objective, and niche based particle swarm optimization algorithms, and single, multi-objective, and niche based bat algorithms.

\subsection{Paper Division}

Section 2 of the paper presents the background of the problem, Section 3 gives the literature review, Section 4 gives the proposed methodology, Section 5 gives the experimentation with results, Section 6 is the discussion section, and finally, Section 7 presents the conclusion and Section 8 presents the future work.

\section{Background}

\subsection{Feature Subset Selection}

The feature subset selection method is also known as dimensionality reduction [20] and/or attribute selection [21]. It selects the most relevant features that result in an increased classification accuracy and better time efficiency due to the decreased number of features.

\subsubsection{Different Approaches for Feature Subset Selection}

\section{Filter Approach}

This feature subset selection method is independent of the used classifier. Filter techniques serve as pre-processing techniques of selecting the subset. Filter methods give emphasis to the finding of relevant associations among features and high-level concepts. Filter techniques make use of straightforward statistics as a result of empirical feature distribution. These empirical distribution methods pick strongly related features while filtering out weakly related features at the evaluation process of overall feature selection process.

\section{Wrapper Approach}

This approach is very simple and dependent on the classifier involved [22]. It includes the accuracy of the classifier as the optimization function, thus it is highly dependent on the classifier used. In this approach, a variety of feature subsets are evolved and then evaluated on the basis of the classification accuracy of the classifier, and the best feature subset is selected.

\section{Embedded Approach}

The embedded approach has a high classifier involvement. The subset search optimizing algorithm is constructed inside the classifier model. Hence, these approaches are highly dependent on the classifier and the search algorithm. In these techniques, the search space and the feature subset space are combined in the search. 


\subsubsection{Optimization Problem}

An optimization probelm is a problem in which the objective is to find a solution in the search space that has the minimum (or the maximum) value of the objective function. It can be categorized as:

\section{Single Objective}

A single objective is a problem that has only one objective to optimize, e.g., in a feature selection problem, it can be the classification accuracy or it can be the number of selected features. The focus is only on the optimization of one single objective at one time.

\section{Multi-Objective}

A multi-objective is a problem that has multiple objectives to optimize simultaneously. One objective could be to maximize while the other could be to minimize at the same time.

\subsubsection{Scalarization}

A multi-objective problem is often solved by combining its multiple objectives into one single-objective scalar function, which makes it very simple to solve. In this case, there is only one optimal solution instead of multiple non-dominated optimal solutions. This approach is known as the weighted-sum or the scalarization method. This paper also used the scalarization method for solving multi-objective optimization through the weighted-sum of classification accuracies and the number of features selected by every individual solution.

$$
f=\Sigma w_{k} f_{k}
$$

where $\mathrm{k}=1: \mathrm{k}, \Sigma \mathrm{w}_{\mathrm{k}}=1$, " $\mathrm{f}$ " represents the fitness function, and $\mathrm{k}$ represents the number of objectives. Different weights are tried for objective types, accuracy, and number of features. The best results are found on weights $w 1=0.7$ and $w 2=0.3$ for accuracy and number of features, respectively.

\subsubsection{Multi-Objective Feature Subset Selection}

Multi-objective feature subset selection selects the minimum number of features from a larger dataset, which improves the accuracy of the classifier and increases the time efficiency, as discussed earlier. Thus, the two main objectives for fitness function are:

- Increase classifier accuracy;

- Decrease number of features.

In multi-objective optimization, there are conflicting objectives; as in the feature subset selection problem, the classification accuracy has to be maximized, and the number of selected features has to be minimized. Optimization of one objective will never provide the best solution, thus there is a need to optimize function and trade-off among all the objectives. The resultant solution will be optimized to give the best results by taking all the objectives into account.

\subsubsection{Genetic Algorithm}

GA [11] is a nature motivated algorithm that fits within a bigger arrangement of evolutionary algorithms. It emulates the conduct of characteristic development and choice procedure by Charles Darwin, i.e., the idea of survival of the fittest. GA is utilized to make a productive answer for search and optimization problems. The principle parts of the genetic algorithm were fundamentally motivated by the regular evolution process, for example, legacy, change, determination, and hybrid are connected on the person to get upgraded arrangements.

The genetic algorithm begins from the introduction of the initial population. The initial population is randomly created and, after that, the process of evolution is connected to the population to produce 
the fittest individual (chromosome as a specialized genetic algorithm). The advancement prepared then proceeds until the stopping criteria (objective function criteria) are met.

\subsubsection{Particle Swarm Optimization (PSO)}

PSO [12] is one of the swarm based intelligent algorithms. The algorithm was inspired by the basis of swarm (of fish and birds) flocking patterns. The algorithm can be understood by a simple example of a bird group in search of food. The bird closest to food has a louder voice, and other birds move towards this bird according to its volume. This particular bird is called the leader of the flock. The leader of the group keeps changing according to the difference of distance from food. The process continues until the leader reaches the food.

\section{Literature Review}

In recent years, a number of approaches have been proposed for solving feature subset selection and other optimization problems using meta-heuristics algorithms such as GA, PSO, BA, etc. This section reviews those approaches that are relevant to the proposed approach of this paper.

Cheng Fu-Tsai et al. proposed a technique that executes a bat algorithm in a parallel manner [23]. It divides the initial population into multiple subpopulations and runs each single population or group on a separate processor. It shares the information at the information sharing phase of the algorithm. At this stage, each subpopulation shares its information with all other subpopulations. It increases the efficiency of the algorithm and makes the convergence faster as the environments get parallelized altogether. Their proposed algorithm was compared with the naive bat algorithm [24] by running them on four benchmark functions; results show that the parallelized bat algorithm outperforms the simple bat algorithm not only in terms of time but also in terms of quality.

This paper [16] proposes an algorithm that uses the echolocation behavior of natural bats to find its solution/prey. Bats fly randomly with velocity ' $v$ ' at position ' $x$ ' with fixed frequency. As the bat approaches its prey, it increases its pulse emission rate while decreasing its loudness. The algorithm was compared with GA and PSO on five benchmark functions, and results show that BA outperforms GA and PSO both in accuracy and efficiency.

This paper [12] presents a feature selection technique that uses PSO and support vector machine (SVM) as a classifier to find the accuracy of selected feature subsets. This method was applied on five different sized benchmark datasets, and results show that the proposed algorithm reduces features while giving high classification accuracy at the same time.

This paper [25] claims to be the first paper on multi-objective feature selection using PSO. It proposes two algorithms to solve the problem. The first approach finds non-dominated solutions using a simple approach to find the Pareto front [25], while the second approach uses mutation, crowding, and dominance to find the Pareto fronts. These two approaches were then compared for single objective and multi-objective feature selection on 12 benchmark datasets, and results show that the first approach outperforms the second approach. This paper proposes an improved PSO in order to perform attribute selection for the task of predicting coronary artery heart disease using the improved fuzzy artificial neural network classifier. A dataset of 7525 diabetic patients was used, out of which 4329 were males and 3196 were females. The research was carried out separately for the male and female patients, and the study achieved maximum accuracies of $88.82 \%$ and $88.05 \%$ for male and female patients, respectively, using improved PSO as an optimization algorithm for attribute selection [26].

This paper proposes instance selection using filter based and wrapper based bat algorithms as well as the cuckoo search algorithm with an application in e-fraud detection. The classifier used was SVM. The proposed techniques were evaluated on 24 datasets. Filter based techniques improved the training speed on all the datasets using SVM while not compromising the accuracy, whereas the wrapper based approaches improved the accuracy on $74 \%$ of the datasets with significant improvement on the training speed of SVM [27]. 
Brits et al. [28] proposed a niching technique with GA. It is a technique used for finding multiple optimal solutions for a problem at the same time, and the concept itself is called multimodal optimization. This paper proposes that niching can be done using two methods that are given below:

\subsection{Fitness Sharing Method}

The fitness sharing strategy rewards individuals that can exceptionally search good zones of the area while profoundly debilitating similar individuals in a space [15]. This is the reason the population differs among itself, which keeps up diversity in the population that ultimately avoids local minima.

\subsection{Swarming Method}

In the swarming strategy, a small amount of the global population indicated by a rate, namely the generation gap, replicates and dies in every iteration of GA [15]. A parent replaces the most comparative individual (as far as genotype correlation) taken from a random sub population of size crowding factor (swarming component) from the global population. The deterministic swarming strategy presents rivalry in the middle of offspring and parents of indistinguishable corners. This system has a tendency to distinguish arrangements with higher fitness and to eliminate solutions with lower fitness.

Gheyas et al. proposed a hybrid algorithm named as SAGA (Simulated Annealing Genetic Algorithm) [1], a name taking into account two meta-heuristic methods, simulated annealing (SA) and GA, that combines various qualities of existing algorithms to select the ideal subsets from an extensive component space. The SAGA algorithm is a hybrid of various wrapper strategies, i.e., an SA, a GA, a GRNN (generalized regression neural network), and a greedy search algorithm. They compared and analyzed the proposed algorithm (SAGA) with the traditional search algorithms (ACO, FW, GA, PSO, SA, SBS, SFBS, SFFS, and SFS) on 30 high dimensional datasets. Their proposed algorithm outperforms other algorithms [1].

This paper [29] proposes a technique that uses the optimum-path forest classifier. It encodes features in binary form where " 0 " represents a feature's absence in the solution, and " 1 " represents a feature's presence in the solution. The optimum-path forest is considered as a speedy classifier, and the exploration power of the bat algorithm combined with the speed of the optimum-path classifier was applied on five public datasets and yielded results that demonstrate that this method performs better than other swarm based algorithms or meta-heuristics.

Niche PSO [28] does not use social information of the swarm. Instead, it randomly distributes the particles into the whole search space, and particles use their cognitive information and perform local searches instead-these particles are called swarms. Every time a particle comes into the range of radius of any other particle, it gets merged into that swarm and forms a bigger swarm. It was applied on five benchmark functions and compared with the niche based genetic algorithm (NGA), and it gives better results as compared to GA in terms of convergence and finding all maxima points.

\section{Proposed Methodology}

This research presents a niche based bat algorithm for feature subset selection and compares its results with single objective and multi objective GA, PSO, and bat algorithms. Comparative analysis of niche based BA was performed with niche based GA and niche based PSO algorithms for performance and efficiency.

\subsection{Bat Algorithm}

Bat Algorithm behaves as a bat, as it incorporates the echolocation behavior of bats. By using this behavior, bats find the location of their prey. Given below is a Bat Algorithm (Algorithm 1) [16]: 


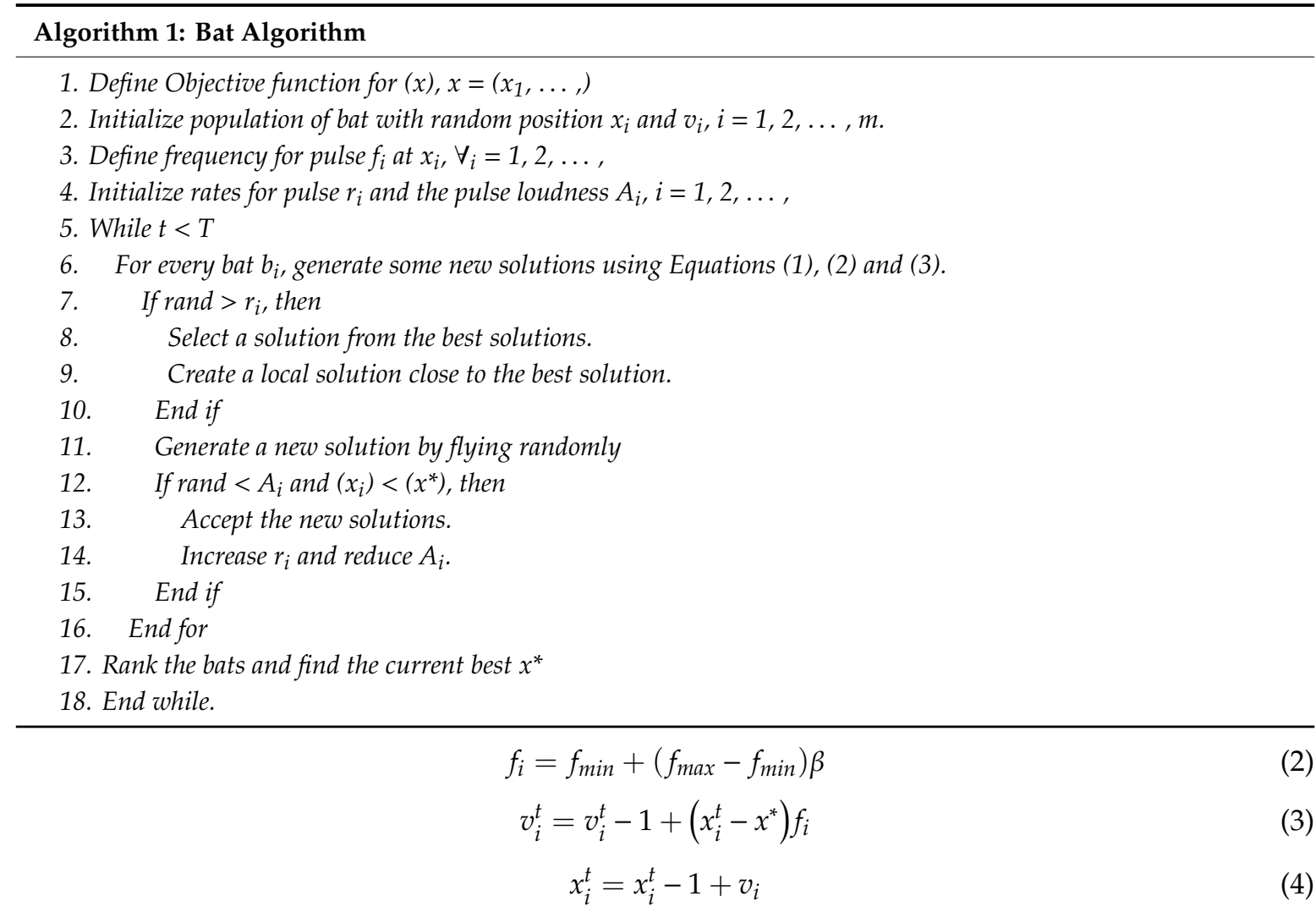

$x^{*}$ is the current global best solution in the population.

$$
x_{\text {new }}=x_{\text {old }}+\varepsilon A^{t}
$$

where $\varepsilon$ belongs to $[-1,1]$ is a random number, while $A^{t}=\left\langle A_{i}^{t}\right\rangle$ is the average loudness of all the bats at this time step. Equation (5) is used for the random walk in line number 11.

\subsection{How Bat Algorithm Works}

In the beginning, every bat is initialized with frequency, loudness, pulse rate, velocity, and the random position in the search space in line numbers 2,3 , and 4 of the algorithm. Later, their positions are updated according to the position of the prey. Frequency decreases as the distance decreases. Equation (2) is used for updating the frequency. The algorithm will run until the maximum number of iterations is reached. To enhance the exploration power of the algorithm, random walk is being performed in line number 11. Then, from lines 12 to 15 , if any one of the local solutions has a better fitness value than the global best solution, it becomes the global best solution, and pulse rate increases and loudness decreases. Finally, after the maximum number of iterations, the solutions are ranked according to the criteria, and the best solution is selected as a global solution in line number 16 of the algorithm.

\subsection{Bat Representation as a Solution for Feature Selection}

Single bat or solution is represented with bits, as shown in Figure 1. The number of bits will be equal to the number of features in the dataset. The first bit represents the first feature, and the second bit represents the second feature in the dataset. The " 0 " bit value shows that this feature has not been selected, and the " 1 " bit value shows the existence of a feature in the selection process. The same bat representation is used as the binary version of GA, PSO, MOBA, MOPSO, MOGA, and NBBA. The binary encoding makes the classifier easy to classify and also improves its accuracy and performance. Binary bat is encoded as: 


\begin{tabular}{|c|c|c|c|c|c|c|}
\hline$F_{0}$ & $F_{1}$ & $F_{2}$ & $F_{3}$ & $F_{4}$ & \multicolumn{2}{|}{} \\
\hline 1 & 0 & 1 & 1 & 0 & $\cdots$ & 1 \\
\hline
\end{tabular}

Figure 1. Solution encoding in bat.

\subsection{Evaluation Function}

The evaluation function used by NBBA is the weighted sum of the classifier's accuracy and the number of features that are selected by the solution. The same function is used for MOBA, MOPSO, and MOGA, but not for GA and PSO, which use the classifier's accuracy or the number of features at any time, as they optimize the single objective problem. The dataset is split into testing and training datasets. The classifier is trained using the training dataset, and the testing dataset shows the accuracy of the classifier. If the bat producing the best fitness value is found, and all the other subsets have been explored, then the algorithm has converged and stops, or the algorithm completes the maximum number of iterations.

\subsection{Classifier Used}

The K-nearest neighbor ( $\mathrm{k}-\mathrm{NN}$ ) was used as a classifier in our proposed technique. This classifier is one of the well-known nonparametric classification methods. The k-NN algorithm is a supervised learning algorithm. The k-NN classifies the input data on the basis of the k-nearest neighbor's category. $\mathrm{k}-\mathrm{NN}$ is used for its simplicity in many research problems, such as in [30], where it is used for the prediction of cancer using gene expression data. In our case, the dataset was continuous (integer/real), thus using k-NN was the best choice for our research, as the paper claims that the performance of $\mathrm{k}-\mathrm{NN}$ on continuous datasets is better than its performance on the text data [31]. It performs equally well with any other statistical machine learning algorithms, as this paper claims [32]. In a recently published paper [33], k-NN was used as a classifier for predicting heart disease with PSO as a feature optimizer, and k-NN predicted heart disease with $100 \%$ accuracy.

\section{Classification Measurement}

To evaluate the algorithm, the classification accuracy was computed using one of the four evaluation metrics that are widely used to compute the performance of the classifier $[4,5]$. The accuracy was computed using the following formula:

$$
\text { Accuracy }=\sum \frac{T P+T N}{T P+T N+F P+F N}
$$

\subsection{Niche Based Bat Algorithm (NBBA), Proposed Algorithm}

The input for the Algorithm 2 is: training set T1 with labels and test set T2, number of features $n$, number of iterations I, population size $m$, loudness $A$, pulse emission rate $r, \alpha$ and $\gamma$ values.

The output consists of: features subset $\mathrm{F}$ that gives the maximum accuracy over T2.

\subsection{How NBBA Works}

The niche based bat algorithm (Algorithm 2) initializes the population similarly to the bat algorithm with additional parameters that include the radius of the sub populations and the global best solution of each sub population. The main population is then trained through random walk using Equation (5) in the beginning of the algorithm and again after each iteration to preserve the diversity among the solutions in the main population. Then, their fitness is updated. Initially, there is a single sub population that is also the main population. To identify more sub populations, the algorithm trains the sub populations with the full bat algorithm [9] and updates the fitness of the bats/solutions and finally updates the radius associated with each sub population. Radius $\left(R_{k}\right)$ defines the boundary of 
the sub population. The radius of the sub population $P_{k}\left(P_{k}\right.$ is the $k$ th sub population) is the distance between the global best solution of that sub population and the solution that is farthest from the global best solution of the similar sub population, which is represented by Equation (6) given below. The solutions in the population are the binary strings. Therefore, Hamming distance measure is used for calculating the distance between the two solutions.

$$
R_{k}=\left(\left\|P_{k} \cdot x_{g}-P_{k} \cdot x_{i}\right\|\right)
$$

$P_{k} \cdot x_{g}$ represents the global/best solution/bat in the sub population $k$, while $P k$. xi represents all other particles in that swarm.

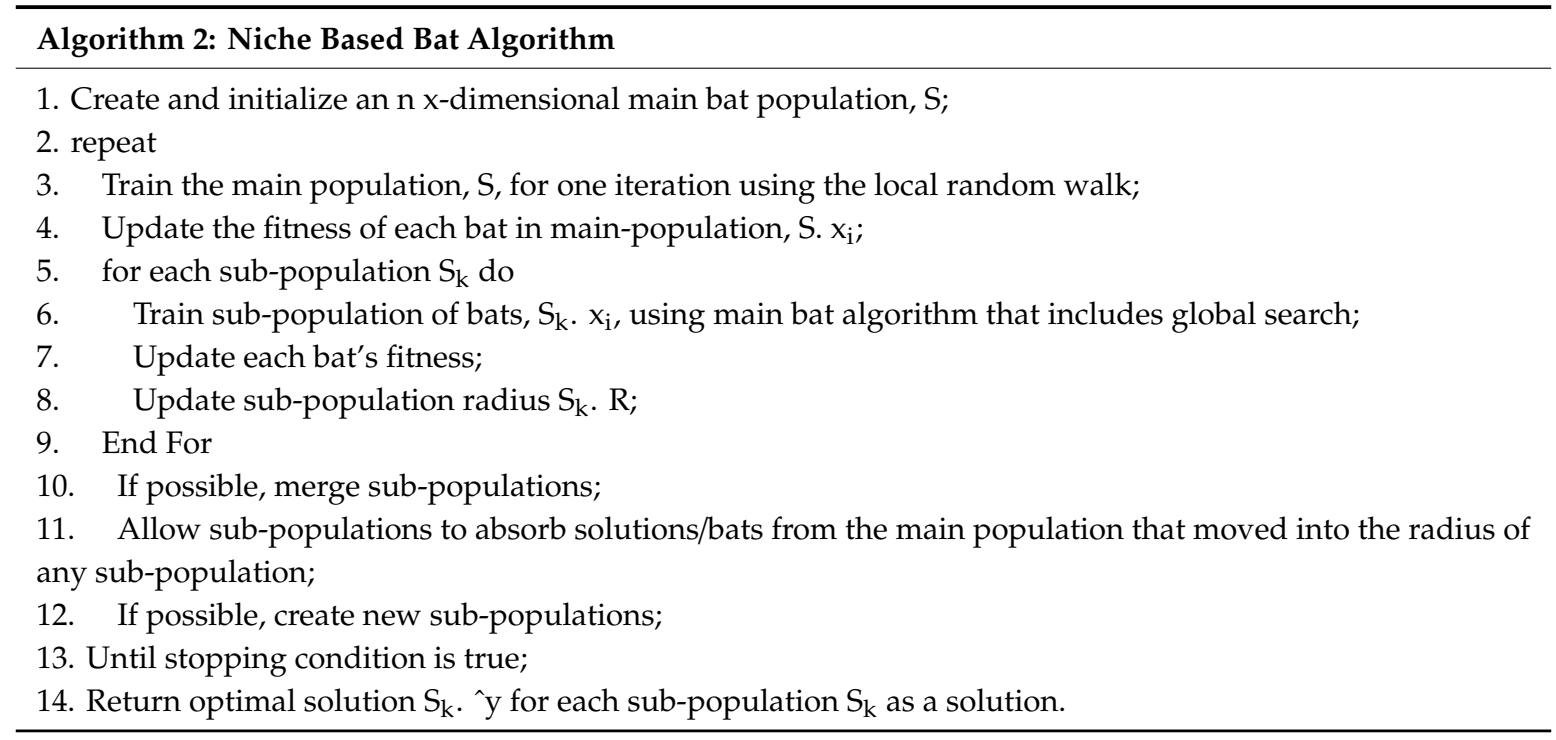

The algorithm merges two sub populations $\left(P_{k 1}\right.$ and $\left.P_{k 2}\right)$ only if possible (line 9$)$ when the distance between their global solutions is less than the sum of their radii, which is given by Equation (7).

$$
\left\|P_{k 1} \cdot x_{g}-P_{k 2} \cdot x_{g}\right\|<\left(R_{k 1}+R_{k 2}\right)
$$

The algorithm moves the particular solution into the sub populations (line 10) when the solutions move into the radius of the sub population that is given by the Equation (8).

$$
\left\|x_{i}-P_{k} \cdot x_{g}\right\|<R_{k}
$$

The algorithm creates new sub populations in (line 11) when a solution in the main population does not change its fitness significantly after a few iterations (in our case, the number was five). Then, the algorithm combines that solution with the nearest solution in the main population that forms a new sub population, and the algorithm also calculates the radius of the new sub population using Equation (6).

Finally, the algorithm runs until the iterations are complete. After that, all the global best solutions from the sub populations are returned by the algorithm, and the solution with the best fitness (the problem is changed to the minimization problem) among the global solutions from each sub population is considered as the optimal solution by the algorithm.

\subsection{Contribution}

This paper presents four main contributions: (i) the developed and proposed NBBA; (ii) the NBBA was used for a feature subset selection problem and can be used along with any other classification 
technique; (iii) use of the niche PSO for feature subset selection problems; (iv) use of the niche GA for feature subset selection problems.

\section{Experimentation and Results}

\subsection{Datasets}

The datasets used for classification purposes were Madelon, ionosphere, and sonar datasets taken from the online UCI machine learning data repository [34]. The used datasets were benchmark datasets for classification as shown in Table 1.

Table 1. Dataset description in detail.

\begin{tabular}{cccccc}
\hline Dataset Name & Total Samples & $\begin{array}{c}\text { No. of } \\
\text { Attributes }\end{array}$ & $\begin{array}{c}\text { Attribute } \\
\text { Characteristics }\end{array}$ & $\begin{array}{c}\text { Train/Test } \\
\text { Ratio }\end{array}$ & No. of Classes \\
\hline Ionosphere & 351 & 34 & integer, real & $70 / 30$ & $2(\mathrm{Good} / \mathrm{Bad})$ \\
Sonar & 208 & 60 & real & $70 / 30$ & $2(\mathrm{Mine} / \mathrm{Rock})$ \\
Madelon & 4400 & 500 & real & $70 / 30$ & $2(+1 /-1)$ \\
\hline
\end{tabular}

\subsection{Results}

The parameter settings, the classification accuracies, and the number of features selected along with the comparative analyses for GA, PSO, BA, MOGA, MOPSO, MOBA, niche GA, niche PSO, and NBBA are given in Tables $2-11$ below: 
Table 2. Parameter Settings for genetic algorithm (GA), multi-objective genetic algorithm (MOGA), single objective niche GA, and multi-objective niche GA.

\begin{tabular}{|c|c|c|c|c|}
\hline Parameters & GA & MOGA & Single Objective Niche GA & Multi-Objective Niche GA \\
\hline Particle representation & Bit string & Bit string & Bit string & Bit string \\
\hline Population & 30 & 50 & 50 & 50 \\
\hline Selection method & Tournament selection & Tournament selection & Tournament selection & Tournament selection \\
\hline Elitism & 0.3 & 0.3 & 0.3 & 0.3 \\
\hline Generation Gap & 0.7 & 0.7 & 0.7 & 0.7 \\
\hline Classifier & k-nearest neighbor (k-NN) & k-nearest neighbor & k-nearest neighbor & k-nearest neighbor \\
\hline Fitness function & k-NN accuracy & $($ w $1 \times$ k-NN accuracy $)+($ w2 $\times$ no. of features $)$ & k-NN accuracy & $(\mathrm{w} 1 \times \mathrm{k}-\mathrm{NN}$ accuracy $)+(\mathrm{w} 2 \times$ no. of features $)$ \\
\hline
\end{tabular}

Table 3. Parameter settings for particle swarm optimization (PSO), multi-objective particle swarm optimization (MOPSO), single objective niche PSO, and multi-objective niche PSO.

\begin{tabular}{|c|c|c|c|c|}
\hline Parameter & PSO & MOPSO & Single Objective Niche PSO & Multi-Objective Niche PSO \\
\hline Particle representation & Bit string & Bit string & Bit string & Bit string \\
\hline Swarm size & 30 & 30 & 50 & 50 \\
\hline Inertia Weight & 0.8 & 0.8 & 0.8 & 0.8 \\
\hline Classifier & k-nearest neighbor & k-nearest neighbor & k-nearest neighbor & k-nearest neighbor \\
\hline Fitness function & k-NN accuracy & $(\mathrm{w} 1 \times \mathrm{k}-\mathrm{NN}$ accuracy $)+(\mathrm{w} 2 \times$ no. of features $)$ & k-NN accuracy & (w1 $1 \times \mathrm{k}-\mathrm{NN}$ accuracy $)+(\mathrm{w} 2 \times$ no. of features $)$ \\
\hline
\end{tabular}

Table 4. Parameter Settings for bat algorithm (BA), multi-objective bat algorithm (MOBA), single objective niche based BA (NBBA), and multi-objective niche based BA.

\begin{tabular}{|c|c|c|c|c|}
\hline Parameter & BA & MOBA & Single Objective NBBA & Multi-Objective NBBA \\
\hline Particle representation & Bit string & Bit string & Bit string & Bit string \\
\hline Population & 30 & 30 & 50 & 50 \\
\hline Loudness & 0.9 & 0.9 & 0.9 & 0.9 \\
\hline Pulse rate & 0.3 & 0.3 & 0.3 & 0.3 \\
\hline $\mathrm{F}_{\min }$ & 0 & 0 & 0 & 0 \\
\hline $\mathrm{F}_{\max }$ & 2 & 2 & 2 & 2 \\
\hline Classifier & k-nearest neighbor & k-nearest neighbor & k-nearest neighbor & k-nearest neighbor \\
\hline Fitness function & k-NN accuracy & $(\mathrm{w} 1 \times \mathrm{k}-\mathrm{NN}$ accuracy $)+(\mathrm{w} 2 \times$ no. of features $)$ & k-NN accuracy & (w1 $1 \times \mathrm{k}-\mathrm{NN}$ accuracy $)+(\mathrm{w} 2 \times$ no. of features $)$ \\
\hline
\end{tabular}


Table 5. Results of single, multi-objective, and niche based genetic algorithm.

\begin{tabular}{ccccc}
\hline & & & Datasets \\
\hline \multirow{2}{*}{ Details } & & Ionosphere & Sonar & Madelon \\
& Instances & 351 & 208 & 4400 \\
Features selected & Features & 34 & 60 & 500 \\
using & GA & 12 & 33 & 265 \\
& MOGA & 10 & 17 & 256 \\
Accuracy & Single-objective Niche GA & 16 & 30 & 287 \\
& Multi-objective Niche GA & 8 & 15 & 196 \\
\hline & k-NN & $80.64 \%$ & $80.00 \%$ & $69.50 \%$ \\
& GA + k-NN & $92.38 \%$ & $91.96 \%$ & $77.83 \%$ \\
& MOGA + k-NN & $91.28 \%$ & $88.70 \%$ & $75.16 \%$ \\
& Multi-objective Niche GA + k-NN & $90.28 \%$ & $91.93 \%$ & $78.50 \%$ \\
\hline
\end{tabular}

Table 6. Results of single, multi-objective, and niche based PSO.

\begin{tabular}{ccccc}
\hline & & & Datasets \\
\hline \multirow{2}{*}{ Details } & & Ionosphere & Sonar & Madelon \\
& Instances & 351 & 351 & 4400 \\
Features selected & Features & 34 & 34 & 500 \\
using & PSO & 16 & 16 & 325 \\
& MOPSO & 9 & 9 & 312 \\
Accuracy & Single-objective Niche PSO & 26 & 26 & 317 \\
& Multi-objective Niche PSO & 9 & 9 & 213 \\
\hline & k-NN & $80.64 \%$ & $80.00 \%$ & $69.50 \%$ \\
& PSO + k-NN & $91.70 \%$ & $91.70 \%$ & $75.80 \%$ \\
\hline & MOPSO + k-NN & $89.52 \%$ & $89.52 \%$ & $76.00 \%$ \\
& Multi-objective Niche PSO + k-NN & $91.42 \%$ & $92.38 \%$ & $76.50 \%$ \\
\hline
\end{tabular}

Table 7. Results of single, multi-objective, and niche based BA.

\begin{tabular}{ccccc}
\hline & & & Datasets & \\
\hline & & Ionosphere & Sonar & Madelon \\
\hline \multirow{2}{*}{ Details } & Instances & 351 & 208 & 4400 \\
& Features & 34 & 60 & 500 \\
\hline \multirow{2}{*}{ Features selected } & BA & 17 & 34 & 253 \\
using & MOBA & 8 & 16 & 225 \\
& Single-objective NBBA & 19 & 34 & 248 \\
Accuracy & Multi-objective NBBA & 6 & 13 & 178 \\
& k-NN & 80.64 & 80.00 & 69.50 \\
& BA + k-NN & 93.33 & 91.93 & 78.33 \\
& MOBA + k-NN & 92.38 & 93.54 & 78.33 \\
\hline
\end{tabular}


Table 8. Comparison of number of features selected by single objective GA, PSO, and BA with their classification accuracies.

\begin{tabular}{ccccccc}
\hline \multirow{2}{*}{ Dataset } & \multicolumn{2}{c}{ GA } & \multicolumn{2}{c}{ PSO } & \multicolumn{2}{c}{ BA } \\
\cline { 2 - 6 } & Features & Accuracy & Features & Accuracy & Features & Accuracy \\
\hline Ionosphere & 12 & $92.38 \%$ & 16 & $91.7 \%$ & 17 & $93.33 \%$ \\
Sonar & 33 & $91.93 \%$ & 36 & $88.70 \%$ & 34 & $91.93 \%$ \\
Madelon & 265 & $77.83 \%$ & 325 & $75.83 \%$ & 253 & $78.33 \%$ \\
\hline
\end{tabular}

Table 9. Comparison of number of features selected by niche based single objective GA, PSO, and BA with their classification accuracies.

\begin{tabular}{ccccccc}
\hline \multirow{2}{*}{ Dataset } & \multicolumn{2}{c}{ GA } & \multicolumn{2}{c}{ PSO } & \multicolumn{2}{c}{ BA } \\
\cline { 2 - 6 } & Features & Accuracy & Features & Accuracy & Features & Accuracy \\
\hline Ionosphere & 16 & $90.28 \%$ & 26 & $92.38 \%$ & 19 & $91.7 \%$ \\
Sonar & 30 & $91.93 \%$ & 36 & 90.32 & 34 & $93.54 \%$ \\
Madelon & 287 & $78.5 \%$ & 317 & $76.5 \%$ & 248 & $79.16 \%$ \\
\hline
\end{tabular}

Table 10. Comparison of number of features selected by multi-objective GA, PSO, and BA with their classification accuracies.

\begin{tabular}{ccccccc}
\hline \multirow{2}{*}{ Dataset } & \multicolumn{2}{c}{ MOGA } & \multicolumn{2}{c}{ MOPSO } & \multicolumn{2}{c}{ MOBA } \\
\cline { 2 - 6 } & Features & Accuracy & Features & Accuracy & Features & Accuracy \\
\hline Ionosphere & 10 & $91.28 \%$ & 9 & $89.52 \%$ & 8 & $92.38 \%$ \\
Sonar & 17 & $88.7 \%$ & 21 & $91.93 \%$ & 16 & $93.54 \%$ \\
Madelon & 256 & $75.16 \%$ & 312 & $76 \%$ & 225 & $78.33 \%$ \\
\hline
\end{tabular}

Table 11. Comparison of number of features selected by niche based multi-objective GA, PSO, and BA with their classification accuracies.

\begin{tabular}{ccccccc}
\hline \multirow{2}{*}{ Dataset } & \multicolumn{2}{c}{ Niche GA } & \multicolumn{2}{c}{ Niche PSO } & \multicolumn{2}{c}{ Niche BA } \\
\cline { 2 - 6 } & Features & Accuracy & Features & Accuracy & Features & Accuracy \\
\hline Ionosphere & 8 & $93.33 \%$ & 9 & $91.42 \%$ & 6 & $93.33 \%$ \\
Sonar & 15 & $91.93 \%$ & 19 & $91.93 \%$ & 13 & $95.16 \%$ \\
Madelon & 196 & $79.16 \%$ & 213 & $76.5 \%$ & 178 & $80.16 \%$ \\
\hline
\end{tabular}

\section{Discussion}

\section{Comparative Analysis}

This paper presents purely empirical research in nature. Details are clearly presented about the datasets along with the parameter settings that were selected after conducting experiments on different parameter settings. The parameters with the best settings are listed in Tables 1-3. Implementation of the algorithms can be provided on demand through email.

The experiments were conducted in four rounds; the mean accuracy and the mean number of features were calculated by using 10-fold cross validation over five runs of the algorithms on each dataset. In the first round, the accuracies on the dataset were calculated as listed in Table 12, using k-NN as a classifier without using any of the algorithms for optimizing the number of features. 
Table 12. Resultant accuracy and number of features using different single objective algorithms.

\begin{tabular}{ccccccc}
\hline & \multicolumn{2}{c}{ Ionosphere } & \multicolumn{2}{c}{ Sonar } & \multicolumn{2}{c}{ Madelon } \\
\hline Algorithm & Features & Accuracy & Features & Accuracy & Features & Accuracy \\
\hline GA & 12 & $92.38 \%$ & 33 & $91.93 \%$ & 265 & $77.83 \%$ \\
Niche GA & 16 & $90.28 \%$ & 16 & $90.28 \%$ & 287 & $78.50 \%$ \\
PSO & 16 & $91.7 \%$ & 36 & $88.70 \%$ & 325 & $75.83 \%$ \\
Niche PSO & 26 & $92.38 \%$ & 36 & 90.32 & 317 & $76.5 \%$ \\
BA & 17 & $93.33 \%$ & 34 & $91.93 \%$ & 253 & $78.33 \%$ \\
Niche BA & 19 & $91.7 \%$ & 34 & $93.54 \%$ & 248 & $79.16 \%$ \\
\hline
\end{tabular}

In the second round of experiments, accuracies were calculated by retrieving the best features using GA, PSO, and BA, as given in Table 13. However, being a single objective (to maximize the classification accuracy) experiment, the number of features selected was not taken into account by the algorithms. BA outperformed GA and PSO on ionosphere and Madelon datasets, while GA outperformed PSO and BA on the sonar dataset.

Table 13. Resultant accuracy and number of features using different multi-objective algorithms.

\begin{tabular}{ccccccc}
\hline & \multicolumn{2}{c}{ Ionosphere } & \multicolumn{2}{c}{ Sonar } & \multicolumn{2}{c}{ Madelon } \\
\hline Algorithm & Features & Accuracy & Features & Accuracy & Features & Accuracy \\
\hline GA & 12 & $92.38 \%$ & 17 & $88.7 \%$ & 256 & $75.16 \%$ \\
Niche GA & 8 & $93.33 \%$ & 15 & $91.93 \%$ & 196 & $79.16 \%$ \\
PSO & 9 & $89.52 \%$ & 21 & $91.93 \%$ & 312 & $76.00 \%$ \\
Niche PSO & 8 & $93.33 \%$ & 19 & $91.93 \%$ & 213 & $76.5 \%$ \\
BA & 8 & $92.38 \%$ & 16 & $93.54 \%$ & 225 & $78.33 \%$ \\
Niche BA & 6 & $93.33 \%$ & 13 & $95.16 \%$ & 178 & $80.16 \%$ \\
\hline
\end{tabular}

In the third round of experiments, the problem was multi-objective in nature, thus the scalarization method was used, as given in Equation (1), with the weight of 0.3 for the number of features and the weight of 0.7 for the classification accuracy. The experiments were performed using different weight values, and the values with the best results are quoted in the table. Table 9 shows that MOBA outperformed MOGA and MOPSO in terms of accuracy and number of features selected on all three datasets.

In the fourth round, the experiments were performed with multi-objective niche GA, niche PSO, and NBBA, and the results (as seen in Table 10) show that multi-objective NBBA outperformed multi-objective niche GA and niche PSO in terms of classification accuracy and number of features selected.

The results clearly indicate that adding the niching technique to the bat algorithm can enhance the diversity in the population, ultimately avoiding the local minima. The results show that NBBA outperforms all the other algorithms, including their different variations as well.

\section{Conclusions}

The proposed algorithm, i.e., NBBA, was compared with niche based GA and niche based PSO. Experiments were carried out to compare the performances of the bat algorithm with the genetic algorithm and the particle swarm optimization algorithm. Implementation results show that single objective and multi-objective bat algorithms yield better results than single and multi-objective genetic algorithm and particle swarm optimization algorithm. Thus, this study presents a direction to explore the bat algorithm with the niching technique. 


\section{Future Work}

\subsection{Advantages of NBBA}

Single and multi-objective NBBA performs better than the other existing algorithms mentioned in this paper, and, due to the strategy the NBBA adopts, we anticipate that it will also perform better than other state of the art algorithms, such as Non-Dominated Sorting Genetic Algorithm (NSGA II) and Strength Pareto Evolutionary Algorithm (SPEA II). NBBA finds non-dominated solutions or the Pareto front and optimizes with the strategy it builds upon, which is provided in the coming paragraph. Basically, NBBA divides the whole search space into multiple regions called niches by incorporating multiple bats in those regions. By doing this, NBBA avoids the local minima problem because random walk of the bats in the main population moves the bats in different regions of the search space in every iteration of the NBBA, which makes it possible for them to be included in different niches/regions of the search space. This enhances the diversity of the solutions in the search space and ultimately avoids the local minima. Bats/solutions in multiple niches trained with the full BA only explore using random walk and exploits using the global best solution in that particular niche. Similarly, bats in other regions of the search space also explore and exploit those regions and find the best solution in only that region of the search space. Ultimately, the algorithm picks the best solution out of the best solutions of every niche [34].

Table 14 shows the comparison of NSGA-II and NBBA for the three datasets used in this paper, i.e., ionosphere, sonar, and Madelon. "Generation" represents the iterations for evolutionary algorithms. The population is the initial set of solutions that begins the optimization problem. These parameters are set, and resultant values of accuracy and number of features selected are reported. The comparison shows the number of features selected with NBBA.

Table 14. Comparison of NSGA-II with NBBA for their classification accuracies on ionosphere, sonar, and Madelon datasets.

\begin{tabular}{ccccccc}
\hline \multirow{2}{*}{ Dataset } & \multicolumn{3}{c}{ NSGA-II } & \multicolumn{2}{c}{ NBBA } \\
\cline { 2 - 6 } & Generation & Population & Features & Accuracy & Features & Accuracy \\
\hline \multirow{2}{*}{ Ionosphere } & 100 & 20 & 2 & 96.59 & \multirow{2}{*}{9} \\
& 50 & 16 & 2 & 96.59 & 63.33 \\
\multirow{2}{*}{ Sonar } & 100 & 20 & 6 & 98.07 & \multirow{2}{*}{9} & 95.16 \\
& 50 & 16 & 12 & 100 & 86.16 \\
\hline \multirow{2}{*}{ Madelon } & 100 & 20 & 149 & 86 & 178 & 8.4 \\
\hline
\end{tabular}

\subsection{Limitations}

This paper applies a scalarization technique that makes a multi-objective problem into a single objective problem and does not make use of the objectives to optimize independently in order to get the true non-dominated or the Pareto optimal solutions. It compares different weights manually in order to find the true Pareto optimal solutions. In the future, the scalarization technique can be replaced, and NBBA can be implemented in such a way that it finds a non-dominated solution set that must be close to the actual or the true Pareto front. To make the future direction more clear, this paper compares the results of the above mentioned single objective and multi-objective NBBA with NSGA II on the same three datasets. The results show that considering objectives independently and finding non-dominated solutions can give better results.

Author Contributions: N.S. developed the theory and implemented the algorithms, while A.F.S. contributed in carrying out the experiments and future work. They wrote the manuscript with the support from K.Z. K.Z. guided N.S. and A.F.S. while supervising and helping shape the research. All authors discussed the results and contributed to the final manuscript. 
Funding: This research received no external funding.

Conflicts of Interest: The authors declare no conflict of interest.

\section{Abbreviations}

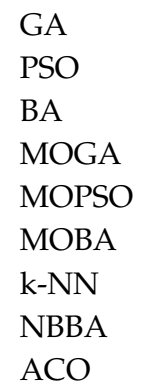

\section{References}

1. Gheyas, I.A.; Smith, L.S. Feature subset selection in large dimensionality domains. Pattern Recognit. 2010, 43, 5-13. [CrossRef]

2. Boopathi, V.; Subramaniyam, S.; Malik, A.; Lee, G.; Manavalan, B.; Yang, D.-C. mACPpred: A Support Vector Machine-Based Meta-Predictor for Identification of Anticancer Peptides. Int. J. Mol. Sci. 2019, $20,1964$. [CrossRef]

3. Chen, Y.-W.; Lin, C.-J. Combining SVMs with Various Feature Selection Strategies. In Feature Extraction; Springer: Berlin/Heidelberg, Germany, 2006; pp. 315-324.

4. Manavalan, B.; Shin, T.H.; Kim, M.O.; Lee, G. PIP-EL: A New Ensemble Learning Method for Improved Proinflammatory Peptide Predictions. Front. Immunol. 2018, 9, 1783. [CrossRef]

5. RSu; Hu, J.; Zou, Q.; Manavalan, B.; Wei, L. Empirical comparison and analysis of web-based cell-penetrating peptide prediction tools. Brief. Bioinform. 2019. [CrossRef]

6. Gulgezen, G.; Cataltepe, Z.; Yu, L. Stable feature selection using MRMR algorithm. In Proceedings of the 2009 IEEE 17th Signal Processing and Communications Applications Conference, Antalya, Turkey, 9-11 April 2009; pp. 596-599.

7. Peng, H.; Long, F.; Ding, C. Feature selection based on mutual information: Criteria of max-dependency, max-relevance, and min-redundancy. IEEE Trans. Pattern Anal. Mach. Intell. 2005, 27, 1226-1238. [CrossRef]

8. Osei-Bryson, K.-M.; Giles, K.; Kositanurit, B. Exploration of a hybrid feature selection algorithm. J. Oper. Res. Soc. 2003, 54, 790-797. [CrossRef]

9. Soufan, O.; Kleftogiannis, D.; Kalnis, P.; Bajic, V.B. DWFS: A wrapper feature selection tool based on a parallel genetic algorithm. PLoS ONE 2015, 10, e0117988. [CrossRef]

10. Engelbrecht, A.P. Computational Intelligence: An Introduction; John Wiley \& Sons: Hoboken, NJ, USA, 2007.

11. Vafaie, H.; de Jong, K. Genetic algorithms as a tool for feature selection in machine learning. In Proceedings of the Fourth International Conference on Tools with Artificial Intelligence TAI '92, Arlington, VA, USA, 10-13 November 1992; pp. 200-203.

12. Tu, C.; Chuang, L.; Chang, J.; Yang, C. Feature selection using PSO-SVM. IAENG Int. J. Comput. Sci. 2007, 33, $1-6$.

13. Sun, Y.; Gao, Y. A Multi-Objective Particle Swarm Optimization Algorithm Based on Gaussian Mutation and an Improved Learning Strategy. Mathematics 2019, 7, 148. [CrossRef]

14. K Nearest Neighbor. Available online: https://en.wikipedia.org/wiki/K-nearest_neighbors_algorithm (accessed on 27 September 2018).

15. Sareni, B.; Krähenbühl, L. Fitness sharing and niching methods revisited. IEEE Trans. Evol. Comput. 1998, 2, 97-106. [CrossRef]

16. Yang, X.-S. A New Metaheuristic Bat-Inspired Algorithm; Springer: Berlin/Heidelberg, Germany, 2010; pp. 65-74.

17. Yang, X.-S. Bat Algorithm: Literature Review and Applications. Int. J. Bio-Inspired Comput. 2013. [CrossRef]

18. Musikapun, P.; Pongcharoen, P. Solving Multi-Stage Multi-Machine Multi-Product Scheduling Problem Using Bat Algorithm. In Proceedings of the 2nd International Conference on Management and Artificial Intelligence, Bangkok, Thailand, 7-8 April 2012; IACSIT Press: Singapore, 2012; Volume 35. 
19. Yadav, S.L.; Phogat, M. A Review on Bat Algorithm. Int. J. Comput. Sci. Eng. 2017, 5, 39-43. [CrossRef]

20. Taha, A.M.; Tang, A.Y.C. Bat Algorithm for Rough Set Attribute Reduction. J. Theor. Appl. Inf. Technol. 2013, $51,10$.

21. An Introduction to Feature Selection. Available online: https://machinelearningmastery.com/an-introductionto-feature-selection/ (accessed on 27 September 2018).

22. Kohavi, R.; John, G.H. Wrappers for feature subset selection. Artif. Intell. 1997, 97, 273-324.

23. Tsai, C.-F.; Dao, T.-K.; Yang, W.-J.; Nguyen, T.-T.; Pan, T.-S. Parallelized Bat Algorithm with a Communication Strategy; Springer: Cham, Switzerland, 2014; pp. 87-95.

24. Taha, A.M.; Mustapha, A.; Chen, S.-D. Naive Bayes-guided bat algorithm for feature selection. Sci. World, J. 2013, 2013, 325973. [CrossRef]

25. Xue, B.; Zhang, M.; Browne, W.N. Particle Swarm Optimization for Feature Selection in Classification: A Multi-Objective Approach. IEEE Trans. Cybern. 2013, 43, 1656-1671. [CrossRef]

26. Narasimhan, B. Altered particle swarm optimization based attribute selection strategy with improved fuzzy Artificial Neural Network classifier for coronary artery heart disease risk prediction. Int. J. Adv. Res. Ideas Innov. Technol. 2019, 5, 1196-1203.

27. AA AKINYELU. On the Performance of Cuckoo Search and Bat Algorithms Based Instance Selection Techniques for SVM Speed Optimization with Application to e-Fraud Detection. KSII Trans. Internet Inf. Syst. 2018, 12. [CrossRef]

28. Brits, R.; Engelbrecht, A.P.; van den Bergh, F. A niching particle swarm optimizer. In Proceedings of the Conference on Simulated Evolution and Learning, Singapore, 1 January 2002; pp. 692-696.

29. Nakamura, R.Y.M.; Pereira, L.A.M.; Costa, K.A.; Rodrigues, D.; Papa, J.P.; Yang, X.-S. BBA: A Binary Bat Algorithm for Feature Selection. In Proceedings of the 25th SIBGRAPI Conference on Graphics, Patterns and Images, Ouro Preto, Brazil, 22-25 August 2012; pp. 291-297.

30. Ayyad, S.M.; Saleh, A.I.; Labib, L.M. Gene expression cancer classification using modified k-nearest neighbors technique. BioSystems 2019, 176, 41-51. [CrossRef]

31. Raikwal, J.S.; Saxena, K. Performance Evaluation of SVM and k-nearest neighbor Algorithm over Medical Data set. Int. J. Comput. Appl. 2012, 50, 35-39.

32. Gunavathi, K.P.C. Performance Analysis of Genetic Algorithm with kNN and SVM for Feature Selection in Tumor Classification. Int. J. Comput. Inf. Eng. 2014, 8, 1491-1497.

33. Ma, J. Prediction of heart disease using k-nearest neighbor and particle swarm optimization. Biomed. Res. 2017, 28, 4154-4158.

34. Deb, K.; Pratap, A.; Agarwal, S.; Meyarivan, T. A fast and elitist multiobjective genetic algorithm: NSGA-II. IEEE Trans. Evol. Comput. 2002, 6, 182-197. [CrossRef] 